\title{
How to select optimal mitigation strategies for natural hazards?
}

\author{
O. Špačková \& D. Straub \\ Engineering Risk Analysis Group, Technische Universität München, Germany
}

A. Rimböck

Bavarian Environment Agency, Germany

\begin{abstract}
The planning of mitigation strategies against (natural) hazards requires finding a balance between the cost of the mitigation and the residual risk. As discussed in this paper, existing approaches for selecting the optimal protection level found in literature and practice exhibit several inconsistencies. We provide a general formulation of the optimization problem and study the implications of budget constraints on the solution. The need for an optimal allocation of resources for risk protection amongst different subsystems (e.g. cities or catchments in the case of flood) with a limited budget is investigated. We examine decision criteria, such as Benefit-Cost Ratio (BCR) or Marginal Costs (MC), which are typically used for measuring the efficiency of the investment and prioritizing the investment opportunities. A numerical example demonstrates the identification of an optimal risk mitigation strategy in five regions for different levels of budget constraint. It compares strategies identified with BCR and MC criteria to the globally optimal solution, demonstrating the limitations of the criteria for identifying optimal risk management decision.
\end{abstract}

\section{INTRODUCTION}

The selection of optimal risk protection measures is a central task in many fields of human activity including natural hazard mitigation. As an example, the work presented in this paper has been motivated by the need for optimizing flood risk measures in Alpine valleys in Bavaria, Germany. The maintenance of existing measures is costly and they often do not meet the actual priorities. The authorities consider implementing a new methodology for economically efficient planning and management of flood protection measures. This goal requires the selection of optimal protection strategies in each of the Alpine valleys, considering potential hazard magnitude, resulting damages and cost of measures.

The methodologies for selecting optimal risk protection strategies in engineering, including natural hazard mitigation or safety of buildings and infrastructure, have been adopted from the socioeconomic theory. Because the methodologies are mostly developed by technicians and engineers, the main focus is typically given to the hazard analysis, while the damage assessment and efficiency evaluation are sidelined (Messner and Meyer, 2005). Additionally, the approaches vary among different fields of application and among different countries and several inconsistencies can be found in practice. These are likely to lead to selections of suboptimal protection strategies and to significant differences in investment efficiency among different fields of hazard protection (Rose et al., 2007).

In this paper, we aim at providing a general formulation of the optimization problem assuming that all costs and risks can be expressed in monetary values (or as utility) and that the main objective is minimizing the sum of risk (i.e. expected damage) and cost (i.e. resources needed for establishing and maintaining the protection measures). The allocation of resources for risk protection is viewed as a system optimization problem: the criteria and constraints are set at the top level (e.g. state) and the actual optimization is performed at the level of the subsystems (regions, towns, catchments) when designing particular protection measures and selecting the optimal protection level.

We start with the formulation of the classic continuous optimization problem at the level of a subsystem in Section 2.1 and expand it to the commonly encountered discrete problem in Section 2.2. In Section 3 we introduce the system problem, where mitigation strategies in several subsystems must be optimized jointly. In case of an unlimited budget, it is sufficient to optimize the protection measures in individual subsystems independently. However, if the budget is limited, the need for an optimal allocation of resources amongst the regions arises. In practice, this can be achieved by applying criteria such as Benefit-Cost Ratio (BCR) or Marginal Costs (MC) that are investigated in Sections 3.1 and 3.2. The 


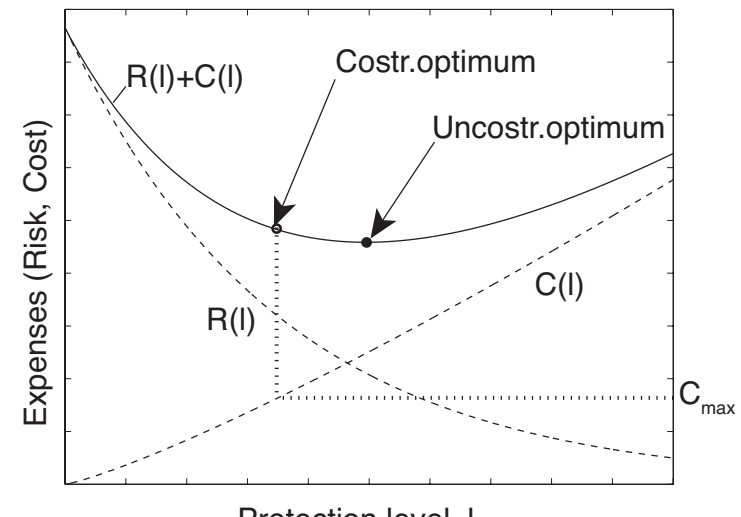

Protection level, I

(a)

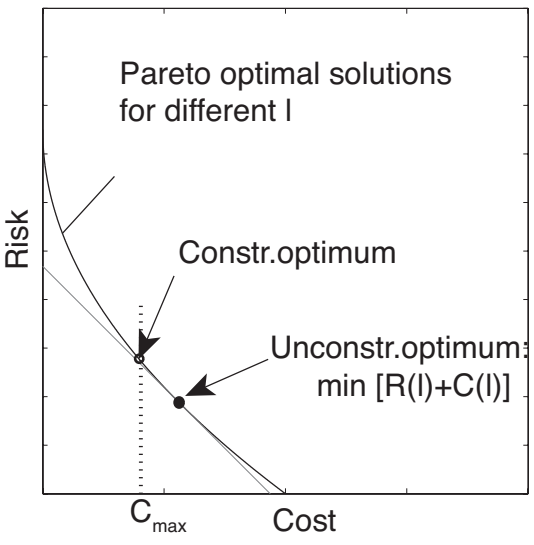

(b)

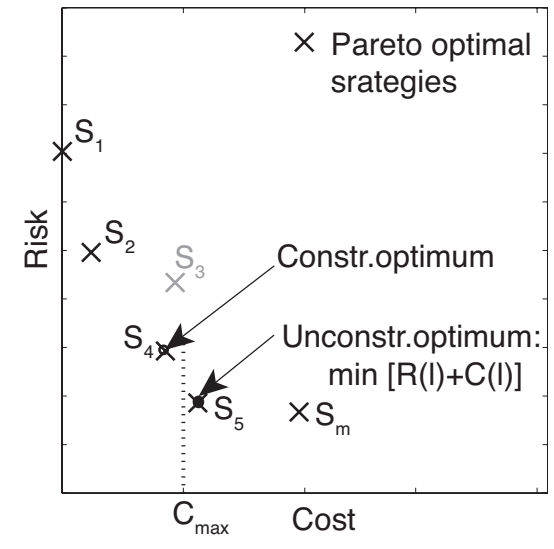

(c)

Figure 1. Alternative illustrations of the risk protection optimization: (a) and (b) continuous formulation, (c) discrete formulation. The figures show unconstrained optimum and the optimum constrained by the available budget $C_{\max }$.

theoretical findings are demonstrated on a hypothetical example of optimizing the protection level in five regions under the constraint of limited budget in Section 4.

\section{OPTIMIZATION OF RISK MITIGATION IN AN INDEPENDENT SUBSYSTEM}

\subsection{Basic continuous formulation}

The optimal risk mitigation strategy is here defined as the one, which minimizes the present (discounted) value of expected monetary expenses over a given period of time. The expenses correspond to the sum of risk, i.e. expected damage caused by the analyzed hazard, and cost, i.e. expected cost for planning, construction, operation and maintenance of the mitigation measures. These two types of expenses are typically incurred by different stakeholders; it is therefore desirable to keep them separately in the analysis. In case of natural hazards, the cost of mitigation measure is part of government investment expenditures, while the risk is covered by special governmental funds, insurance companies and public.

Commonly, the optimization problem is formulated in terms of a protection level $l$. The optimal protection level is found by minimizing:

$\min _{l}[R(l)+C(l)]$

where $R(l)$ and $C(l)$ are the present (discounted) values of the risk and cost, respectively. $C(l)$ is the cost associated with the cheapest mitigation strategy leading to protection level $l$. The problem can be additionally constrained by a maximal available budget $C_{\text {max }}$. Then the objective function from Eq. (1) is subjected to $C(l) \leq C_{\max }$.

Two alternative illustrations of the continuous optimization are shown in Figure 1 (a) and (b). The solid line in both figures represents a set of Pareto optimal solutions for different protection levels. A Pareto optimal solution can in this case be interpret- ed as a state, where given protection level is achieved with minimal possible costs.

An example of a continuous optimization problem is the selection of an optimal height of a coastal dyke (Danzig, 1956).

\subsection{Basic discrete formulation}

In most practical problems, one selects the optimal solution from a countable number of possible risk mitigation strategies and one thus has to solve a discrete optimization problem as is illustrated in Figure 1(c). Such an approach is necessary in cases, where the dependence of risk and cost on the protection level cannot be defined analytically. This situation typically arises when the system consists of many different components (e.g. dykes combined with retention areas, mobile flood barriers and warning system) and each component has one or more optimization parameters (height of a dyke, volume of the reretention, type of the mobile barriers etc.). In such cases, it is only realistic to evaluate the optimum among a countable number of protection strategies, which are identified by the engineers.

Let $S_{1}, S_{2} \ldots S_{m}$ denote the possible strategies. In analogy to Eq. (1), we are searching for the strategy, which minimizes the sum of risk and cost. The optimization problem is now formulated as

$\min _{j}\left[R\left(S_{j}\right)+C\left(S_{j}\right)\right]$

where $R\left(S_{j}\right)$ and $C\left(S_{j}\right)$ are the present discounted values of the expected risk and cost of the $j$ th strategy. The optimization of Eq. (2) can be additionally constrained by the available budget, i.e. it may be subjected to $C\left(S_{j}\right) \leq C_{\max }$.

The strategies displayed in Figure 1(c) with the black crosses represent Pareto optimal solutions, while the gray strategy denoted as $S_{3}$ is not Pareto optimal, because it has higher risk and higher cost than strategy $S_{4}$. 


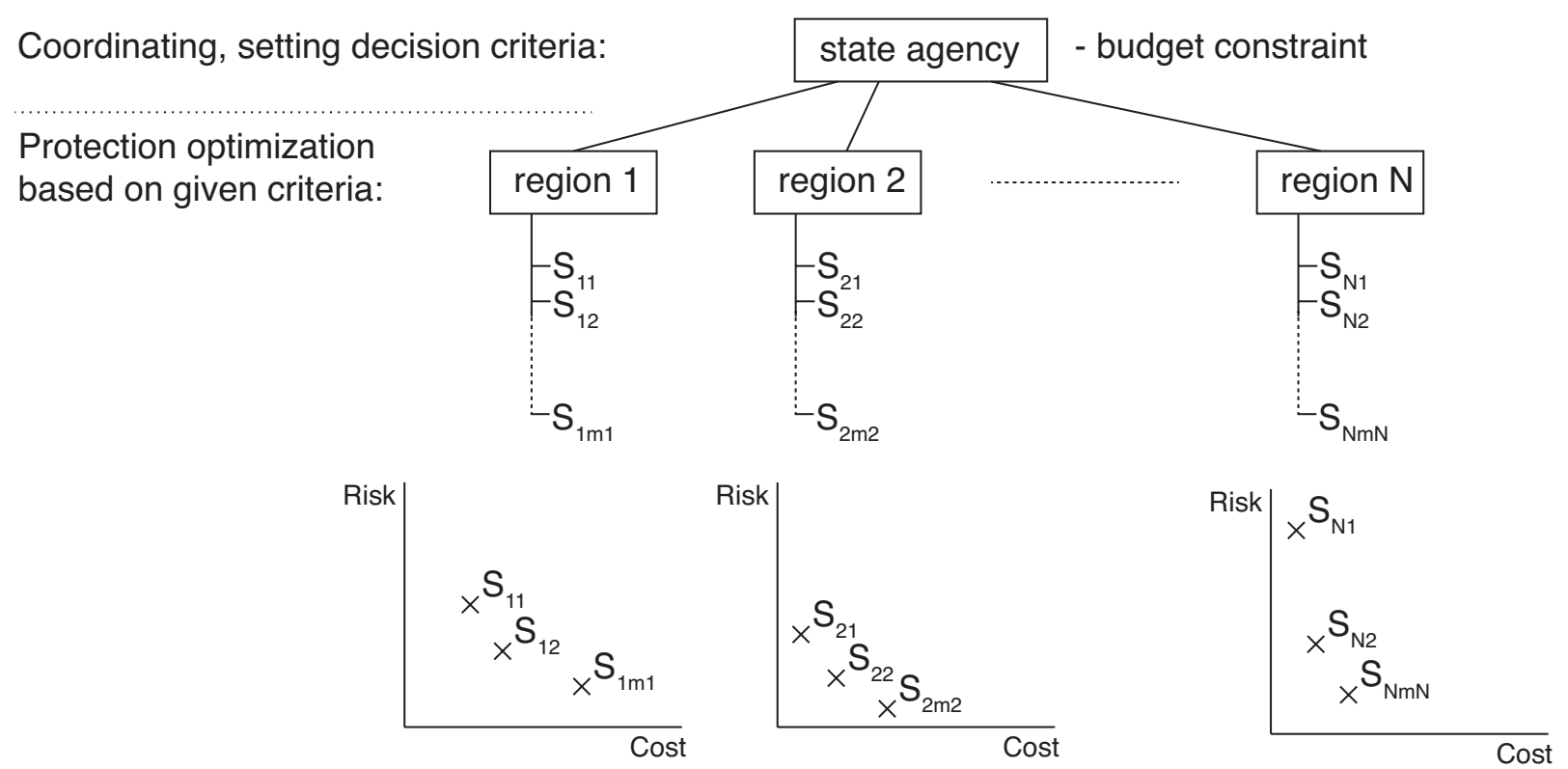

Figure 2. Illustration of the system optimization problem. In each subsystem, Pareto optimal risk mitigation strategies are shown.

\section{HIERARCHICAL OPTIMIZATION AT THE SYSTEM LEVEL}

Risk mitigation measures are often optimized at a system or portfolio level. In flood risk management, an agency can be responsible for various regions (subsystems), which may be catchments or municipalities. The situation is illustrated in Figure 2. In each of the region, an optimal protection strategy must be selected. The optimality is assessed at the system level (from the point of view of the agency), and the optimal solution must ensure an optimal distribution of resources among the subsystems.

In each of $N$ regions, one can identify a number of locally Pareto optimal risk protection strategies denoted as $S_{i j_{i}}$, where $i \in\{1, \ldots, N\}$ is the index of the region, $j_{i} \in\left\{1, \ldots, m_{i}\right\}$ is the index of the strategy in the $i$ th region and $m_{i}$ is the number of strategies in the $i$ th region. Optimization can now be formulated as:

$\min _{j_{1}, j_{2}, \ldots j_{N}} \sum_{i=1}^{N}\left[R\left(S_{i j_{i}}\right)+C\left(S_{i j_{i}}\right)\right]$

where $R\left(S_{i j_{i}}\right)$ and $C\left(S_{i j_{i}}\right)$ are the present (discounted) values of the expected risk and cost of the $j$ th strategy in the $i$ th region.

If the problem of Eq. (3) is unconstrained, the optimal solution can be found by simply finding the optimum in each of the region individually. It holds:

$\min _{j_{1}, j_{2}, \ldots j_{N}} \sum_{i=1}^{N}\left[R\left(S_{i j_{i}}\right)+C\left(S_{i j_{i}}\right)\right]=$

$\sum_{i=1}^{N} \min _{j_{1}, j_{2}, \ldots j_{N}}\left[R\left(S_{i j_{i}}\right)+C\left(S_{i j_{i}}\right)\right]$

However, the cost are typically constrained with the available budget $C_{\max }$ and the objective function from Eq. (3) is subjected to $\sum_{i=1}^{N} C\left(S_{i j_{i}}\right) \leq C_{\max }$. In such a case, Eq. (4) does not hold and the protection cannot be optimized independently in the individual regions. Because the protection strategies in the subsystems are not design at the same time, it is typical- ly impossible to optimize the risk protection measures in the whole system (e.g. in a country or a state) at once.

The problem can be solved by techniques developed for the so-called hierarchical (multi-level) optimization (Stoilov and Stoilova, 2008). In hierarchical optimization, the coordinator (agency) sets criteria (coordination parameters) for the optimization in the subsystems. In case of natural hazard protection, criteria such as Marginal Costs (MC) or Benefit-Cost Ration (BCR) described in detail in Sections 3.1 and 3.2 can be perceived as such coordination parameters.

The hierarchical optimization can be carried out iteratively: The agency can adjust the criteria depending on the results of optimizations in individual subsystems and depending on the changing constraints such as availability of resources.

\subsection{Benefit-cost ratio}

The Benefit-Cost Ratio (BCR) is used for evaluation of natural hazard protection in many countries, see e.g. Defra UK (2010) or Bründl (2009). The BCR of the $j$ th strategy is calculated as

$B C R\left(S_{j}\right)=-\frac{\Delta R\left(S_{j}\right)}{\Delta C\left(S_{j}\right)}$

where $-\Delta R\left(S_{j}\right)$ is the expected value of risk reduction and $\Delta C\left(S_{j}\right)$ is the expected value of cost increase of strategy $S_{j}$ with respect to some reference strategy. The optimal strategy is selected at the subsystem level by maximizing the BCR

$\max _{j} B C R\left(S_{j}\right)=\max _{j}\left[\frac{-\Delta R\left(S_{j}\right)}{\Delta C\left(S_{j}\right)}\right]$

The optimization can be constrained by requiring a minimal value of BCR, i.e. $B C R\left(S_{j}\right) \geq B C R_{\text {req }}$. 
The definition of the reference strategy differs among countries and fields of application. It can be defined as maintaining the currently implemented protection measures, or as a so-called "do-nothing option" or "null option", which corresponds to no active intervention (incl. no maintenance of existing measures).

Here we consider the "null option" as the reference strategy. The BCR then equals:

$\operatorname{BCR}\left(S_{j}\right)=\frac{R_{0}-R\left(S_{j}\right)}{C\left(S_{j}\right)-C_{0}}=\frac{R_{0}-R\left(S_{j}\right)}{C\left(S_{j}\right)}$

where $R\left(S_{j}\right)$ and $C\left(S_{j}\right)$ are the present values of risk and cost of the $j$ th strategy. $R_{O}$ is the maximal level of risk corresponding to the "null option" and the cost of the "null option" is $C_{0}=0$.

Maximizing the BCR does not lead to the optimal solution in the general case. Assuming that the Pareto optimal border has a shape similar to the one shown in Figure 1(b), maximizing the BCR would select a solution, which is very close to the "null" option (i.e. a cheap solution with low protection level). This fact can be better explained on the discrete case shown in Figure 1(c). The BCR criterion identifies strategy $S_{2}$ as the optimum because it has the highest rate or risk reduction to cost from all the options. (Note that strategy $S_{1}$ corresponds to the "null option".) Strategy $S_{2}$ is optimal for small budgets. However, when the available budget exceeds the cost of $S_{4}, S_{2}$ is not the optimal variant anymore and the BCR criterion thus leads to a suboptimal solution. The BCR approach can identify the optimal protection strategies only under restricting conditions: First, the solutions with low protection level are not considered in the optimization (i.e. no strategies with costs lower than $S_{2}$ are included); they are not identified by the analyst or they are discarded by other constraints such as minimal safety levels. Second, the solutions close to the unconstrained optimum (strategies $S_{4}$ and $S_{5}$ ) are not attainable due to the limited budget. The application of the BCR criterion will be shown later in the example in Section 4.

\subsection{Marginal cost criterion}

An alternative approach to risk protection optimization is provided by the marginal cost (MC) criterion. It has been applied in the field of natural hazard protection in Switzerland (Bohnenblust and Troxler, 1987; Bohnenblust and Slovic, 1998; Bründl, 2009). In other fields of engineering risk mitigation, the $\mathrm{MC}$ criterion is not commonly utilized ( $\mathrm{Li}$ et al., 2009).

The marginal costs $\delta C$ are the costs incurred for reducing the risk by an additional unit $|\delta R|$. The principle of the $\mathrm{MC}$ criterion is illustrated in Figure 3 . In the continuous case, the marginal costs can be computed as the derivative of the Pareto optimal costs with respect to the risk, i.e. they can be found graphically as the gradient of the tangent to the Pareto front. If the marginal costs are higher than the risk reduction, i,e. $\delta C>|\delta R|$, the strategy is inefficient (e.g. strategy $S_{j+2}$ in Figure 3). If we require $\delta C=|\delta R|$, the criterion leads to the unconstrained optimum according to Eq. (2).

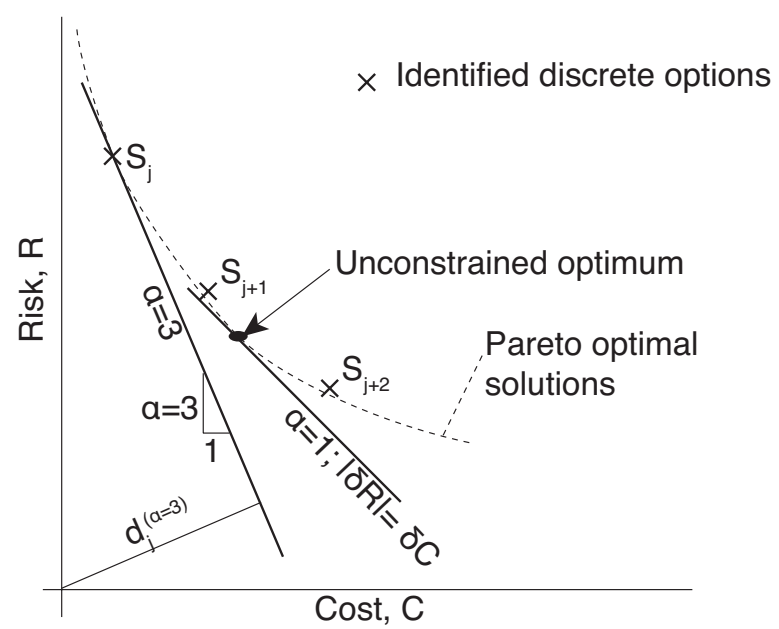

Figure 3. Selection of the optimal strategy with the marginal cost (MC) criterion.

If the budget is limited and we cannot reach the unconstrained optimum, the optimal solution will have $\delta C<|\delta R|$, e.g. strategies $S_{j}$ and $S_{j+1}$ in Figure 3 ..

We can define a value $\alpha>1$, which represents the required efficiency of the investment. The required efficiency is the inverse of the maximally accepted marginal cost $M C_{\max }, \alpha=1 / M C_{\max }$. Then we search for the solution where $\alpha \times \delta C=|\delta R|$.

In the discrete case, or when the optimal solution cannot be found analytically, the optimum can be identified graphically by shifting the line with the gradient corresponding to the required $\alpha$ from the origin to the right (see Figure 3). The optimal solution is the one, which is first reached by this line. For example for $\alpha=3$, the optimal solution corresponds to the strategy $S_{j}$. Computationally, this can be implemented by finding the strategy whose distance $d_{j}^{\alpha}$ (see Figure 3 ) is minimal for given $\alpha$ :

$$
\min _{j} d_{j}^{\alpha}=\frac{1}{\sqrt{\alpha^{2}+1}} \min _{j}\left[\alpha C\left(S_{\mathrm{j}}\right)+R\left(S_{\mathrm{j}}\right)\right]
$$

where $R\left(S_{j}\right)$ and $C\left(S_{j}\right)$ are the present values of the risk and cost of the $j$ th strategy.

Figure 4 illustrates the differences in the selected protection level depending on the utilized criterion. The unconstrained optimum is found with the MC criterion with $\alpha=1$. To find an optimum subjected to budget constraints, the MC criterion with $\alpha>1$ can be used. Under some conditions, as discussed in Section 3.1, also the BCR criterion is suitable for constrained hierarchical optimization. 


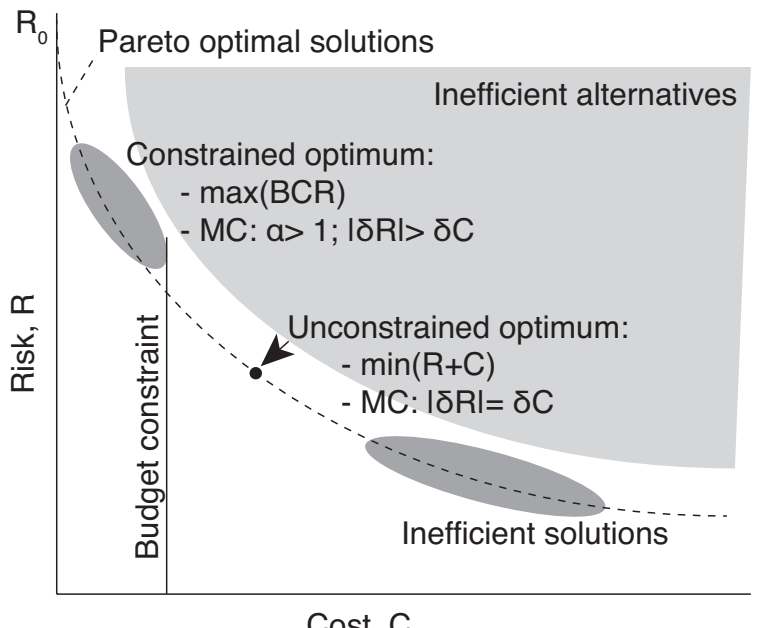

Cost, C

Figure 4. Selection of optimal risk protection level based on different criteria: BCR - Benefit-Cost Ration, MC - marginal costs and minimizing the sum of Risk and Cost. $R_{0}$ is the maximal risk corresponding to a "null" option.

\section{NUMERICAL EXAMPLE}

In this numerical study, we consider the identification of an optimal risk mitigation strategy in $N=5$ regions/subsystems with limited budget. This is motivated by the optimization of flood risk protection measures in Alpine regions, where these regions correspond to individual valleys, catchments or municipalities. In each region, the following is evaluated through engineering studies: the present value of the maximal potential risk $R_{0}$ in case that no measures are taken ("null" option), the present value of risk and cost of the current strategy (current level of protection) and the present value of risk and cost of three to four alternative mitigation strategies. They are summarized in Table 1 . These numbers are hypothetical, but they are similar to real numbers obtained during the planning of flood risk protection in the considered Alpine regions.

Table 1. Risk $\mathrm{R}\left(S_{i j}\right)$ and cost $C\left(S_{i j}\right)$ of alternative strategies in five studied regions ( $\times 10^{5}$ Euro).

\begin{tabular}{llllllll}
\hline Strategy & & Region: & $\mathrm{i}=1$ & $\mathrm{i}=2$ & $\mathrm{i}=3$ & $\mathrm{i}=4$ & $\mathrm{i}=5$ \\
\hline "Null” (N) & $\mathrm{j}=1$ & $R_{0}$ & 8 & 56 & 84 & 13 & 29 \\
& & $C_{0}$ & 0 & 0 & 0 & 0 & 0 \\
Current state & $\mathrm{j}=2$ & $\mathrm{R}\left(S_{i j}\right)$ & 5 & 30 & 32 & 12.5 & 15 \\
$\begin{array}{l}\text { (P) } \\
\text { Option A }\end{array}$ & $\mathrm{j}=3$ & $\mathrm{C}\left(S_{i j}\right)$ & 2 & 10 & 30 & 1 & 10 \\
& & $\mathrm{C}\left(S_{i j}\right)$ & 6 & 39 & 50 & 12 & 22 \\
Option B & $\mathrm{j}=4$ & $\mathrm{C}\left(S_{i j}\right)$ & 1.3 & 5 & 10 & 1.4 & 5 \\
& & $\mathrm{C}\left(S_{i j}\right)$ & 1.8 & 28 & 42 & 10 & 10 \\
Option C & $\mathrm{j}=5$ & $\mathrm{R}\left(S_{i j}\right)$ & 4 & 22 & 20 & 7.5 & 5 \\
& & $\mathrm{C}\left(S_{i j}\right)$ & 2 & 14 & 42 & 3 & 12 \\
Option D & $\mathrm{j}=6$ & $\mathrm{R}\left(S_{i j}\right)$ & 3 & 20 & 17 & 5 & - \\
& & $\mathrm{C}\left(S_{i j}\right)$ & 3 & 15 & 50 & 5 & - \\
\hline
\end{tabular}

The risk protection in all the regions is financed from one budget $C_{\max }$. Following Eq. (3), we aim to select one strategy in each region to minimize the sum of the present value of risk and costs over all regions subject to $\sum_{i=1}^{N} C\left(S_{i j_{i}}\right) \leq C_{\max }$.
Three approaches to the optimization are compared: (I) The complete solution is obtained by testing all the possible combinations for all the regions at once, by excluding those which do not fulfill the budget constraint and by selecting the one with minimal sum of risk and costs according to Eq. (3). The optimization is carried out for different budget levels $C_{\max }=\{2,4,6, \ldots 62\} \times 10^{6}$ Euro. (II) Selecting the optimal solution in each region separately by maximizing the BCR criterion following Eq. (6) subject to $B C R\left(S_{j}\right) \geq B C R_{\text {req }}$, for different levels of $B C R_{\text {req }}$. If none of the options in the given region fulfills $B C R\left(S_{j}\right) \geq B C R_{\text {req }}$, the null option is selected. (III) Selecting the optimal solution in each region separately using the marginal cost criterion following Eq. (8) for different levels of $\alpha$.

Figure 5 and Tables 2-4 display the results of the optimization. Figure 5 shows the total residual risk for all five areas that can be achieved with different levels of budget, as identified using solutions I to III. All identified solutions are Pareto optimal. The unconstrained optimum (with unlimited budget) can be achieved with costs of $44 \times 10^{6}$ Euro and the residual risk is $84 \times 10^{6}$ Euro. It corresponds to selecting options $\mathrm{C}$ in regions 1 and 5 , options $\mathrm{D}$ in regions 2 and 4 and option $\mathrm{A}$ in region 3.

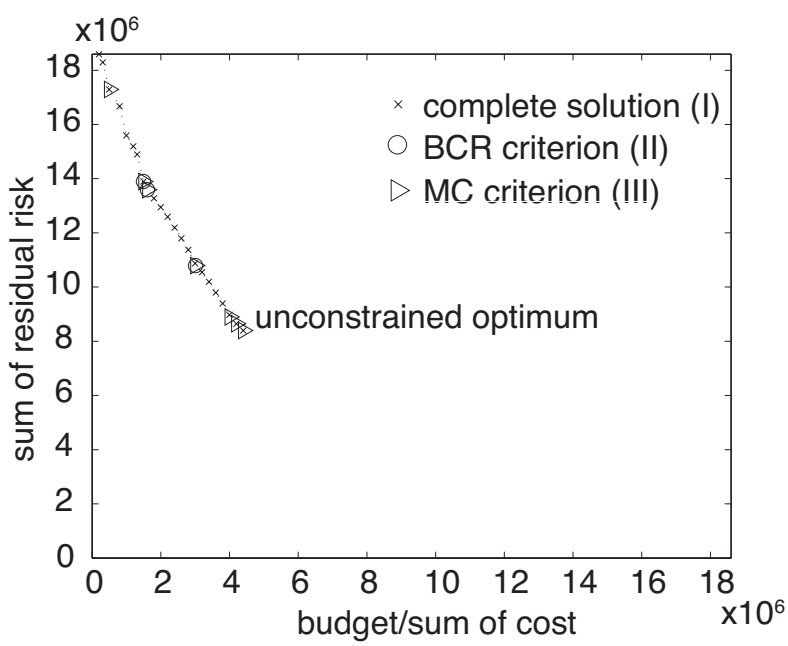

Figure 5. Results of the optimization: Residual risk in all five areas for different levels of budget $C_{\max }$. Comparison of complete solution (I) with solution obtained with BCR criterion (II) and with MC criterion (III).

Tables 2-4 summarize the strategies selected for different levels of budget. $\mathrm{N}$ denotes the "Null" variant, $\mathrm{P}$ denotes the present state and A,B,C and D the other options (compare with Table 1).

Because we select only from a limited number of strategies, it is not possible to always find a solution that fully uses the available budget (see Table 2). For example, there is no combination of strategies that would cost exactly $14 \times 10^{6}$ Euro, we can only find the nearest cheaper one, which costs $13.1 \times 10^{6}$ Euro. 
Table 2. Results of the optimization - complete solution: strategies selected in individual regions for different levels of budget, the corresponding cost $\mathrm{C}$, residual risk $\mathrm{R}$, risk reduction relative to null variant Rred and Net Present Value $(\mathrm{NPV}=\mathrm{R}+\mathrm{C}),\left[\mathrm{x} 10^{6}\right.$ Euro $]$

\begin{tabular}{llllll}
\hline Budget & Strategies & C & R & Rred & NPV \\
\hline 0 & N,N,N,N,N & 0 & 190 & 0 & 190 \\
2 & C,N,N,N,N & 2 & 186 & 4 & 188 \\
4 & C,N,N,B,N & 3.1 & 183 & 7 & 186.1 \\
6 & N,A,N,N,N & 5 & 173 & 17 & 178 \\
8 & B,A,N,B,A & 8 & 166.8 & 23.2 & 174.8 \\
10 & N,N,A,N,N & 10 & 156 & 34 & 166 \\
12 & C,N,A,N,N & 12 & 152 & 38 & 164 \\
14 & C,N,A,B,N & 13.1 & 149 & 41 & 162.1 \\
16 & N,A,A,N,N & 15 & 139 & 51 & 154 \\
18 & B,A,A,B,N & 18 & 132.8 & 57.2 & 150.8 \\
20 & C,A,A,C,N & 20 & 129.5 & 60.5 & 149.5 \\
22 & C,P,A,N,N & 22 & 126 & 64 & 148 \\
24 & N,C,A,N,N & 24 & 122 & 68 & 146 \\
26 & C,C,A,N,N & 26 & 118 & 72 & 144 \\
28 & B,D,A,B,N & 28 & 113.8 & 76.2 & 141.8 \\
30 & B,A,A,B,C & 30 & 108.8 & 81.2 & 138.8 \\
32 & C,A,A,C,C & 32 & 105.5 & 84.5 & 137.5 \\
34 & C,P,A,N,C & 34 & 102 & 88 & 136 \\
36 & N,C,A,N,C & 36 & 98 & 92 & 134 \\
38 & C,C,A,N,C & 38 & 94 & 96 & 132 \\
40 & B,D,A,B,C & 40 & 89.8 & 100.2 & 129.8 \\
42 & C,D,A,C,C & 42 & 86.5 & 103.5 & 128.5 \\
44 & C,D,A,D,C & 44 & 84 & 106 & 128 \\
\hline
\end{tabular}

Table 3. Results of the optimization using BCR criterion: strategies selected in individual regions for different required $B C R_{\text {req }}$, the corresponding cost $\mathrm{C}$, residual risk $\mathrm{R}$, risk reduction relative to null variant Rred and Net Present Value $(\mathrm{NPV}=\mathrm{R}+\mathrm{C}),\left[\mathrm{x} 10^{6}\right.$ Euro]

\begin{tabular}{llllll}
\hline$B C R_{\text {req }}$ & Strategies & $\mathrm{C}$ & $\mathrm{R}$ & Rred & $\mathrm{NPV}$ \\
\hline$>3.5$ & N,N,N,N,N & 0 & 190 & 0 & 190 \\
$2.8-3.4$ & N,A,A,N,N & 15 & 139 & 51 & 154 \\
$2.1-2.7$ & N,A,A,B,N & 16.1 & 136 & 54 & 152.1 \\
$1.0-2.0$ & C,A,A,B,C & 30.1 & 108 & 82 & 138.1 \\
\hline
\end{tabular}

Table 4. Results of the optimization using MC criterion: strategies selected in individual regions for different required $\alpha$, the corresponding $\operatorname{cost} \mathrm{C}$, residual risk $\mathrm{R}$, risk reduction relative to null variant Rred and Net Present Value $(\mathrm{NPV}=\mathrm{R}+\mathrm{C}),\left[\times 10^{6}\right.$ Euro]

\begin{tabular}{llllll}
\hline$B C R_{\text {req }}$ & Strategies & $\mathrm{C}$ & $\mathrm{R}$ & Rred & $\mathrm{NPV}$ \\
\hline$>3.5$ & N,N,N,N,N & 0 & 190 & 0 & 190 \\
3.4 & N,A,N,N,N & 5 & 173 & 17 & 178 \\
$2.8-3.3$ & N,A,A,N,N & 15 & 139 & 51 & 154 \\
$2.0-2.7$ & N,A,A,B,N & 16.1 & 136 & 54 & 152.1 \\
1.9 & C,A,A,B,C & 30.1 & 108 & 82 & 138.1 \\
$1.4-1.8$ & C,D,A,B,C & 40.1 & 89 & 101 & 129.1 \\
1.3 & C,D,A,C,C & 42 & 86.5 & 103.5 & 128.5 \\
$1.0-1.2$ & C,D,A,D,C & 44 & 84 & 106 & 128 \\
\hline
\end{tabular}

As is evident from the results, the BCR approach (II) and the MC approach (III) identify only a subset of possible Pareto optimal strategies. For most budget levels, they do not allow to fully exploit the available budget. For example, they are unable to identify a combination of strategies with total cost between $5 \times 10^{6}$ Euro and $15 \times 10^{6}$ Euro or with total cost between $16.1 \times 10^{6}$ Euro and $30.1 \times 10^{6}$ Euro, even if these exist as shown by approach (I).
Additionally, the BCR criterion (II) only identifies optima with available budgets between $15 \times 10^{6}$ Euro and $30.1 \times 10^{6}$ Euro; in this range it gives the same solutions as the MC criterion (III). However, the BCR criterion is not suitable for higher budgets and for identifying the unconstrained optimum.

\section{SUMMARY \& DISCUSSION}

The formulation of the optimization problem provided in this paper aims at supporting rational decision making for risk protection. The investigated hierarchical risk-based optimization problems arise when decisions on protection measures are taken on the local level (e.g. selection of earthquake mitigation measures of individual bridges, planning of maintenance of offshore oil platforms), while the general aims and budget constraints are given at a top level (e.g. by the bridge management authority or by the company management).

The continuous formulation of the optimization problem presented in Section 2.1 is the one widely used in theory. Figure 1 (a) and (b) show an alternative illustration of the same problem; (a) is typically used in the structural reliability field while (b) is more common in flood risk management. The discrete formulation presented in Section 2.2 describes the problem as it is typically encountered in risk management, which is, however, not commonly formalized in a rigorous way.

Section 2 describes optimization of an independent (sub)system, for example of the flood risk protection of a municipality, where the budget limit is set directly by the local authorities and transfer of resources to other regions or other types of investments is not considered. However, in many applications, the individual subsystems have to compete for a limited amount of resources distributed from a higher level (e.g. state) as described in Section 3. Because the optimization of all subsystems cannot be carried out at once, it is useful to set so-called coordination parameters on the top level, which set a new objective for the individual subsystems. In the long term, the values of the parameters for the selected solution are returned from the subsystems to the top level and based on this information, the coordination parameters can be further adjusted to reflect the actual availability of resources.

The BCR and MC criteria are typically used as such coordinating parameters. Section 3.1 and 3.2 discussed the definition and utilization of these criteria. It was shown that the utilization of the BCR criterion as defined in Eq. (7) for this purpose has fundamental flaws. The BCR criterion works only for a limited range of budget constraint under some restricting conditions (e.g. only in the discrete case). Additionally, in practice the BCR criterion is often defined differently from the definition used here, 
which leads to further, potentially severe, inconsistencies.

The utilization of the MC criterion as a coordination parameter is - in principle - correct. However, in the discrete case the MC criterion might not be sufficiently flexible in order to be able to entirely allocate different levels of budget, as was demonstrated in the numerical example in Section 4. Additionally, unlike in the case of the BCR, it is impossible in the discrete case to determine one exact value of the $\mathrm{MC}$ criterion for the selected solution; one can only provide an interval depending on the neighboring identified Pareto optimal strategies. It is probably for this reason that the $\mathrm{MC}$ criterion is not broadly used in practice, because the interval as an indicator is not as easily interpretable as a single number like the one provided by BCR. Nevertheless, the MC is preferable to the BCR and should be used in practice whenever possible. The MC has the additional advantage over the BCR that it is not necessary to compute the risk $R_{0}$ associated with the reference solution.

In practice, the budget limit is commonly not the only constraint in the optimization problem. Additional constraints are the minimum acceptable safety level and other requirements defined by politics and society. These additional constraints can be included in the process described in this paper simply by excluding the unacceptable strategies. However, even if some of the strategies are inacceptable for safety, political or other reasons, it can be beneficial to quantify their economic efficiency. Knowledge of the $\mathrm{MC}$ of these inacceptable alternatives can help in a long term strategic planning and in improving the regulations and policies.

Here, we have not further investigated how the selection of the MC criterion (the required value of $\alpha)$ at the top level can be implemented. In practice, the iterative process of adjusting the values of the coordination parameters is often limited, because one cannot always flexibly change the protection level in the subsystems once the protection measures have been put in place. It is therefore necessary to have some information on potential risk mitigation strategies in the subsystems when deciding on the value of the coordination parameter.

The paper does not discuss the calculation of risk (i.e. expected damage) and cost. We have implied that their correct value is known. In engineering problems, risk and cost are typically determined by assessing the direct tangible damages and costs. Additional effects, such as potential benefits from future developments in the endangered areas are not included in the analysis. Neglecting these effects can, however, lead to suboptimal decisions, as the societal value of the protection measures will be underestimated (Brent, 1996; Messner and Meyer, 2005). Finally, the impact of uncertainty in estimates of risk and cost on the optimal selection of the mitigation measures should be studied.

\section{CONLUSIONS}

The paper provided a general formulation of the optimization problem for selecting the economically optimal level of risk protection. The optimum was defined as a state minimizing the sum of risk and cost. The types of the problem can be categorized into continuous or discrete optimization. It must be further distinguished whether we optimize a closed subsystem, which is independent from other possible subsystems, or whether we deal with a system problem, where the different subsystems interact because they share a limited budget. The ability of the benefit-cost-ratio (BCR) and the marginal cost (MC) criteria to support an optimal allocation of resources to subsystems were investigated. A numerical example demonstrates the optimization of the discrete system problem and it reveals the limitations of BCR and MC criteria.

\section{ACKNOWLEDGMENT}

The work has been carried out within the project "development of guidelines for Integrated torrent development concepts" financed by the Bavarian Environment Agency.

\section{REFERENCES}

Bohnenblust, H., Slovic, P., 1998. Integrating technical analysis and public values in risk-based decision making. Reliability Engineering and System Safety 59, 151-159.

Bohnenblust, H., Troxler, C., 1987. Risk analysis - Is it a useful tool for the politician in making decisions on avalanche safety?, in: Proceedings of the Davos Symposium. IAHS Publ.

Brent, R.J., 1996. Applied Cost-Benefit Analysis. E. Elgar Pub.

Bründl, M., 2009. Risikokonzept für Naturgefahren - Leitfaden (Risk concept for natural hazards - the Guidelines), Strategie Naturgefahren Schweiz: PLANAT - Swiss National Plattform for Natural Hazards.

Danzig, D. van, 1956. Economic decision problems for flood prevention. Econometrica, J. of the Economic Society 24, 276-287.

Defra UK, 2010. Flood and coastal erosion risk management appraisal guidance. Environment Agency, Bristol, UK.

Li, J., Pollard, S., Kendall, G., Soane, E., Davies, G., 2009. Optimising risk reduction: An expected utility approach for marginal risk reduction during regulatory decision making. Reliability Engineering \& System Safety 94, 1729-1734.

Messner, F., Meyer, V., 2005. Flood damage, vulnerability and risk perception - challenges for flood damage research. UFZ-Diskussionspapiere ( No. 13/2005).

Rose, A., Porter, K., Dash, N., Bouabid, J., Huyck, C., Whitehead, J., Shaw, D., Eguchi, R., Taylor, C., McLane, T., Tobin, L.T., Ganderton, P.T., Godschalk, D., Kiremidjian, A.S., Tierney, K., West, C.T., 2007. Benefit-Cost Analysis of FEMA Hazard Mitigation Grants. Natural Hazards Review 8, 97-111.

Stoilov, T., Stoilova, K., 2008. Goal and predictive coordination in two level hierarchical systems. International Journal of General Systems 37, 181-213. 